\title{
Importance of Surface Preparation for Corrosion Protection of Automobiles
}

\author{
Narayan Chandra Debnath \\ Department of Physics, Institute of Chemical Technology (ICT), Mumbai, India. \\ Email: ndebnath5@rediffmail.com
}

Received December $5^{\text {th }}, 2012$; revised January $7^{\text {th }}, 2013$; accepted January $14^{\text {th }}, 2013$

\begin{abstract}
An overview of science and technology of pretreatment process suitable for automotive finishing with cathodic electrodeposition primer is presented in details in this paper. Both the theoretical principles and practical aspects of tricationic phosphating process that are used in automotive industry are discussed in details. The characteristic features of phosphate coatings of both conventional high zinc phosphating formulations and modern tricationic phosphating formulations on steel surface are compared in details by SEM, EDX and XRD techniques. The corrosion protections of the phosphated and painted steel panels were evaluated by both salt spray test and electrochemical impedance spectroscopy (EIS). The analysis of impedance data in terms of pore resistance (Rpo), coating capacitance (Cc) and breakpoint frequency $\left(\mathrm{f}_{\mathrm{b}}\right)$ as a function of salt spray exposure time provides a clear insight into the mechanism of superior corrosion resistance provided by the modern tricationic phosphating formulations compared with conventional high zinc phosphating formulations.
\end{abstract}

Keywords: Pretreatment Process; Tricationic Phosphating Formulations; SEM; XRD; EDX; Electrochemical Impedance Spectroscopy; Corrosion Protection

\section{Introduction}

The importance of surface preparation for corrosion protection of automobiles need not be over emphasized because the durability of the phosphated and painted metal surface depends quite critically on the quality of cleaning, stabilization of cleaned surface and physicochemical characteristics of the phosphate coating that is deposited on clean surface by chemical conversion process prior to painting of the car body. Industrial surface preparation process generally consists of five processing zones viz: degreasing, derusting, surface activation, phosphating and passivation and these pretreatment chemicals may be used either in spray mode, dip mode or in spray cum dip mode. Modern car manufacturing plants mostly use spray cum full dip mode for its obvious advantage for ensuring satisfactory cleaning and deposition of uniform phosphate coating in the areas of car body which are not normally accessible by spray mode of application. If the car body consists of mixed metal combination for different parts of auto body like mild steel and coated steel, then the in-line derusting stage is eliminated from the pretreatment line. Since the phosphate coating is deposited on a metals surface as a result of interfacial reaction between the metal surface and the phosphating solution, the surface composition of the steel and the method of cleaning will have considerable effect on the structure, composition and morphology of phosphate coating which in turn will affect the final corrosion resistance of the phosphated and painted systems. The structure and composition of the phosphate coating and also its rate of growth depends broadly on the three following factors:

- Structure of the clean metal surface i.e. microstructure and chemical composition of the surface.

- Design of the phosphating and other chemicals used in different pretreatment stages.

- Parameters of the processing baths viz: temperature, concentration, pressure and time of reaction etc.

- The quality of water used for bath preparation and in the rinsing stages after different stages of processing.

The automotive finishing technology has undergone significant changes in the past decades because of demand for car with higher corrosion resistance and better quality of surface finishes [1-16]. The key factors that contributed significantly to the improvement of higher corrosion resistance are the development of new substrates with better inherent corrosion resistance and higher strength, introduction of cathodic electrophoretic paints for priming the car body and development of low temperature tri-cationic phosphating formulation $\left(45^{\circ} \mathrm{C}\right.$ 
to $50^{\circ} \mathrm{C}$ ) which are suitable for depositing excellent phosphate coating on multi metal autobody system containing steel, coated steel and aluminium alloys. The main characteristic features of modern tri-cationic phosphating formulations containing ions of $\mathrm{Zn}, \mathrm{Mn}, \mathrm{Ni}$ is the superior alkali resistance of the resulting phosphate coating which make these formulations highly suitable for operation in cathodic ED bath. This superior alkali resistance of phosphate coating results from the development of additional crystal phases like Phosphophyllite $\left(\mathrm{Zn}_{2} \mathrm{Fe}\left(\mathrm{PO}_{4}\right)_{2} \cdot 4 \mathrm{H}_{2} \mathrm{O}\right)$, Phosphomagnellite $\left(\mathrm{Zn}_{2} \mathrm{Mn}\left(\mathrm{PO}_{4}\right)_{2} \cdot 4 \mathrm{H}_{2} \mathrm{O}\right)$ and Phosphonicolite $\left(\mathrm{Zn}_{2} \mathrm{Ni}\left(\mathrm{PO}_{4}\right)_{2} \cdot 4 \mathrm{H}_{2} \mathrm{O}\right)$ in the coating besides the Hopeite $\left(\mathrm{Zn}_{3}\left(\mathrm{PO}_{4}\right)_{2} \cdot 4 \mathrm{H}_{2} \mathrm{O}\right)$ phase. The higher the value of $\mathrm{P} / \mathrm{P}+\mathrm{H}$ ratio, better is the alkali resistance of the phosphate coating in CED bath leading to superior corrosion resistance of the phosphated and electropainted auto body system [1]. Here, "P" stands for the total Phosphophyllite phases and " $\mathrm{H}$ " stands for Hopeite phase present in the deposited coating. The basic chemical reactions on steel surface in a phosphating bath are described below:

$$
\begin{aligned}
& \mathrm{Fe}+2 \mathrm{H}_{3} \mathrm{PO}_{4} \rightarrow \mathrm{Fe}\left(\mathrm{H}_{2} \mathrm{PO}_{4}\right)_{2}+\mathrm{H}_{2} \\
& 2 \mathrm{Zn}\left(\mathrm{H}_{2} \mathrm{PO}_{4}\right)_{2}+\mathrm{Fe}\left(\mathrm{H}_{2} \mathrm{PO}_{4}\right)_{2}+4 \mathrm{H}_{2} \mathrm{O} \\
& \rightarrow \mathrm{Zn}_{2} \mathrm{Fe}\left(\mathrm{PO}_{4}\right)_{2} \cdot 4 \mathrm{H}_{2} \mathrm{O}+4 \mathrm{H}_{3} \mathrm{PO}_{4} \\
& \text { (Coating-Phosphophyllite) } \\
& \begin{array}{l}
3 \mathrm{Zn}\left(\mathrm{H}_{2} \mathrm{PO}_{4}\right)_{2}+4 \mathrm{H}_{2} \mathrm{O} \\
\rightarrow \mathrm{Zn}_{3}\left(\mathrm{PO}_{4}\right)_{2} \cdot 4 \mathrm{H}_{2} \mathrm{O}+4 \mathrm{H}_{3} \mathrm{PO}_{4}
\end{array} \\
& \text { (Coating-Hopeite) } \\
& 2 \mathrm{Fe}\left(\mathrm{H}_{2} \mathrm{PO}_{4}\right)_{2}+(\mathrm{O}) \\
& \rightarrow 2 \mathrm{FePO}_{4}+2 \mathrm{H}_{3} \mathrm{PO}_{4}+\text { Sludge } \mathrm{H}_{2} \mathrm{O}
\end{aligned}
$$

The coating deposited on steel surface consists of two phases viz: Phosphophyllite and Hopeite as described above. And the sludge, which is a byproduct of the phosphating reaction, settles down on the bottom of the phosphating bath [10-13].

In this work, we discuss in the details the science and technological aspects of a modern tri-cationic phosphateing process which is suitable for deposition of excellent phosphate coating on multi-metal auto body assembly consisting of steel and coated steel and also compatible with cathodic electrodeposition (CED) primers. A large number of experimental techniques like SEM/EDX, XPS, $\mathrm{XRD}$ and AAS, have been used in this work to characterize the chemical composition of steel surface used by automotive manufacturers and also to characterize the morphology, chemical composition, phase composition and coating weight of the phosphate coating deposited on steel surface. Electro chemical impedance spectroscopy (EIS) and Salts spray tests (ASTM B117) have been used for evaluation of overall corrosion resistance of the phosphated and painted steel surface as a function of time and for understanding the underlying mechanism of protection and degradation of the coating system on steel surface over extended period of exposure to corrosive atmosphere.

\section{Pretreatment Process Sequence Used in a Modern Automotive Finishing Plant}

The outline of a 14 stages pretreatment line used in autotive finishing plant is shown in Figure 1. It may be noted that the derusting stage along with post derusting rinse stages have been eliminated from this line because the car body processed in this PT line has a mixed metal combination of steel and electrogalvanised steel in diffrent parts. It may be noted that a combination of spray and dip rinse stages makes effective cleaning between different stages and minimizes the carry over of chemicals to the next stage. The processing parameters of different stages of pretreatment plant are summarized in Table 1. However, under laboratory conditions, the pretreatment process can be implemented in five litre baths and generally the steel panels of $6 " \times 4$ " are used for depositing phosphate coating which can be used for different physico-chemical characterization viz: morphology, phase analysis, chemical analysis and coating weight determination and evaluation of corrosion resistance (ASTM B117) after depositing paint coating of appropriate thickness.

The design of the chemicals used at prephosphating stages viz. degreasing, derusting and surface activation and the corresponding bath parameters all will have considerable effect on the uniformity, morphology, coating weight and quality of phosphate coating deposited during the phosphating stage [17-19]. The physical structure and chemical composition of the phosphate coating, in turn, will affect the corrosion resistance of the phosphated and painted system. Thus it is very important to maintain the bath parameters at the recommended values at every stage of processing to get the right quality of phosphate coating.

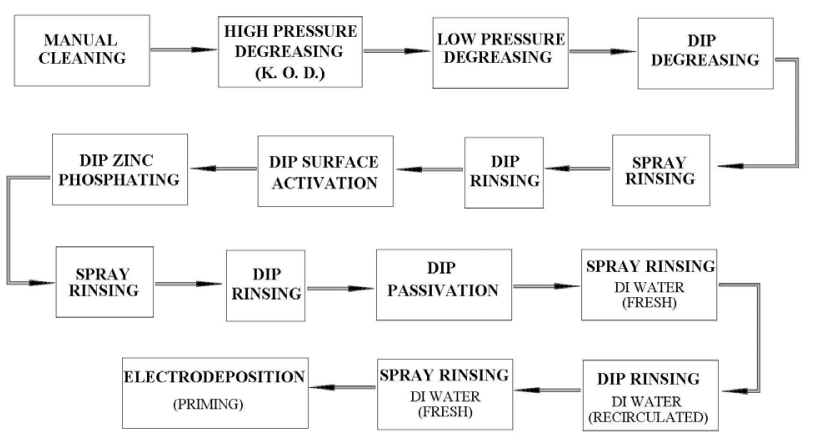

Figure 1. Pretreatment process sequence used in a modern automotive finishing plant. 
Table 1. A 14-stage pretreatment process for automotive finishing.

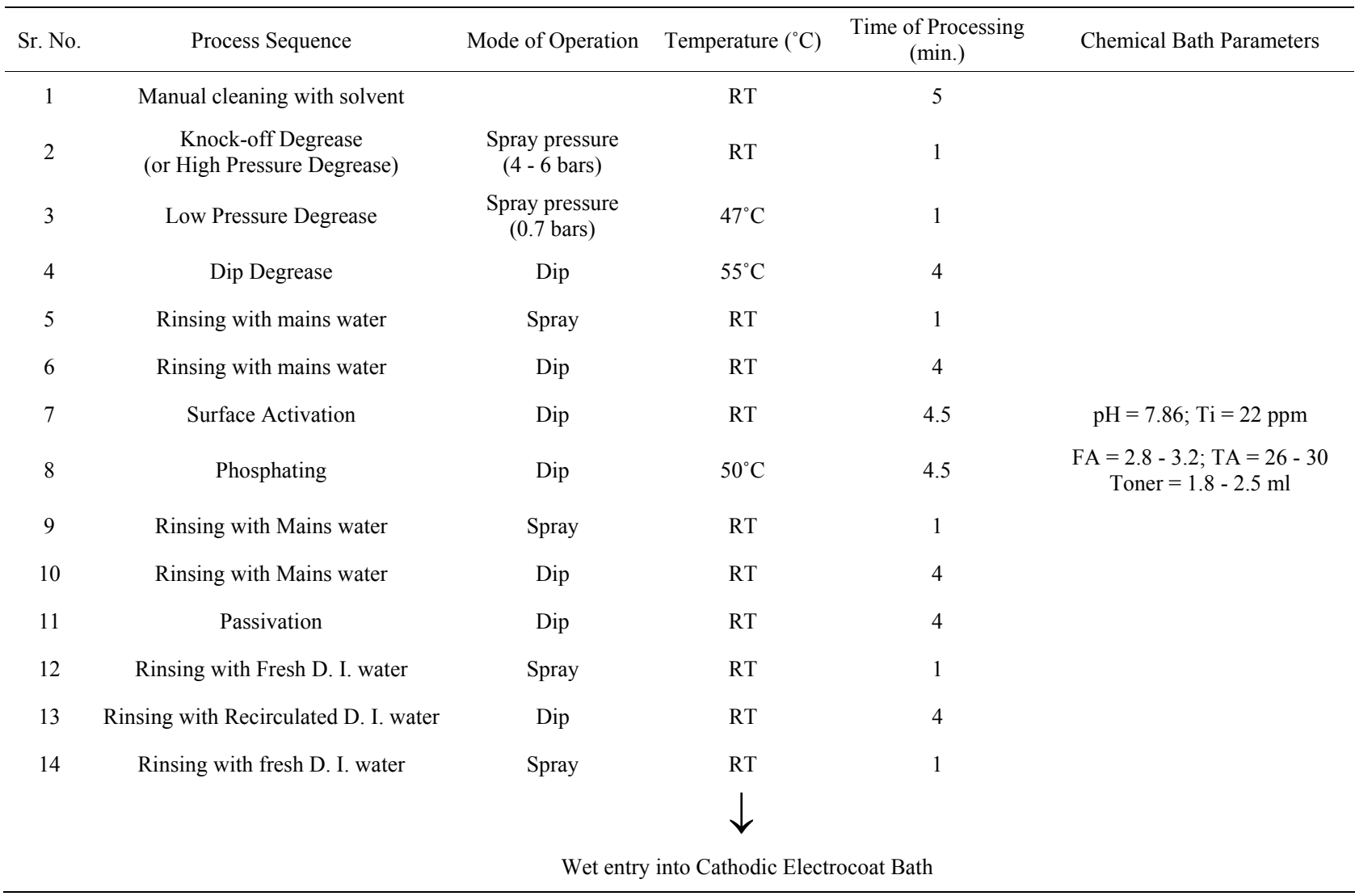

Apart from chemicals, the water quality used in bath make-up as well as at rinse stages plays a very critical role in maintaining the stability of the different baths as well as the quality of the phosphate coating. The post passivation rinse stage is very critical in pretreatment process because soluble salts of chloride, sulfate and ammonia if not removed thoroughly from car body, will promote blistering under a paint film. In order to minimize this possibility, water supply should be free from harmful salts as far as practical. Normally, deionised water (DI) is used for bath make up and the replenishments in surface activation stage, passivation stage and post passivation rinse stages. For other treatment stages like decreasing, derusting, phosphating and other rinse stages mains water may be used provided it conforms to the specification given in Table 2 [7-12]. To ensure minimum carryover of harmful ions to the subsequent stage of CED bath the phosphated surface after passivetion stage is given two or three DI water rinses. Fresh water is used in the last stage, whereas recirculated water is used in first two stages. The conductivity of recirculated water should not exceed $25 \mu \mathrm{S} / \mathrm{cm}$ [1]. The rinse bath should be discarded once the conductivity exceeds the limit of $25 \mu \mathrm{S} / \mathrm{cm}$.

In order to ensure consistently good quality of cleaning and phosphating of car body the following factors in different stages are very important.

\subsection{Degreasing Stage}

The degreasing zone normally consists of at least two stages. The first stage is usually a spray stage known as knock-off-degrease (K.O.D.) and that is followed by a dip stage. The advantage of having two stages is that major portion of the oil, dirt etc. will be removed by high pressure spray impact in the first stage leaving relatively lower load for the dip stage to clean. In Figure 1, the degreasing zone consists of three stages viz. two spray stages and one dip stage for efficient cleaning of car body. In order to ensure maximum efficacy of degreasing stage, misting spray should be provided between K.O.D. and dip degreasing stage and also between dip degreasing and next rinsing stage to prevent the drying of the car bodies during transition from one stage to the next stage. Continuous oil separating systems should also be installed for high volume production of pretreatment line.

\subsection{Surface Activation Stage}

The purpose of this stage is to refine the crystal size of zinc phosphate coating and to control the coating weight during phosphating stage. Modern surface activation che- 
Table 2. Water specification for bath make-up and rinse stages.

\begin{tabular}{|c|c|c|}
\hline Water Type used in PT line & Specifications & Usage \\
\hline I. De-ionised (DI) water & $\begin{array}{l}\text { (i) Conductivity } 5 \mu \mathrm{s} / \mathrm{cm} \\
\text { (ii) } \mathrm{pH} 6.5-7.2\end{array}$ & $\begin{array}{c}\text { Surface activation stage passivation stage post passivation } \\
\text { rinse stage (spray stage) }\end{array}$ \\
\hline $\begin{array}{l}\text { II. Recirculated Deionised } \\
\text { water }\end{array}$ & Conductivity $\leq 25 \mu \mathrm{s} / \mathrm{cm}$ & Post passivation rinse stage (dip stage) \\
\hline \multirow[t]{2}{*}{ III. Mains water } & $\begin{array}{l}\text { 1) Total chloride and sulphate } 70 \mathrm{ppm} \text { maximum } \\
\text { (calculated as } \mathrm{Cl}^{-}+\mathrm{SO}_{4}^{-2} \text { ) } \\
\text { 2) Total alkalinity- } 200 \mathrm{ppm} \text { max. (calculated as } \mathrm{CaCO}_{3} \text { ) }\end{array}$ & \multirow[t]{2}{*}{$\begin{array}{c}\text { Degreasing, derusting stage phosphating stage all other } \\
\text { rinse stages }\end{array}$} \\
\hline & 3) Both together should not exceed $225 \mathrm{ppm}$ & \\
\hline
\end{tabular}

micals are weakly alkaline colloidal dispersion of titanium complex. This treatment leads to the formation of large number of finer crystallites of titanium compound on the metal surface which act as crystal nuclei for the growth of fine zinc phosphate crystals during the phosphating stage. Greater is the number of nucleating centres on the surface, more will be the inter-crystalline collisions and consequently finer will be the crystals and more compact will be the phosphate coating and better will be the paint adhesion and corrosion resistance of the phosphated and painted system $[14,19]$. The efficacy of surface activation bath is critically dependent upon:

- $\mathrm{pH}$ of the bath.

- The concentration of titanium.

- For better colloidal stability of the surface activation bath, it must be made with deionised (DI) water and the activation bath should have a circulation rate of 3 to 4 tank turn-over/hour.

- According to the results published by Yoshihara [1]. The ideal $\mathrm{pH}$ of the activation bath should be in the range of 8.5 to 9.5 and $\mathrm{Ti}$ concentration should be minimum $10 \mathrm{ppm}$. We have observed that within the range of 10 to $30 \mathrm{ppm}$, the coating weight remains essentially constant, Table 1 shows the Ti concentration in the bath as $22 \mathrm{ppm}$.

- The grain refining action depends on amount of Ti adsorbed on the metal surface and for steel surface the adsorption is inversely proportional to the amount of segregated carbon on the surface.

\subsection{Phosphating Stage}

For most effective functioning of modern tricationic zinc phosphating formulation the following points are very important:

- The coating formation reaction is largely dependent upon the free acid (FA), total acid (TA), concentration of oxidizing agents or toners, temperature, deposition time etc. In Table 1 the phosphating bath parameters are given. The phosphate coating weight on mild steel substrates generally lies in the range of 2.8 to $3.2 \mathrm{~g} / \mathrm{m}^{2}$.

- The circulation of phosphating bath solution is an essential requirement for ensuring the deposition of uniform phosphate coating and normally a circulation rate of 3 to 4 tank turn-over/hour is recommended. The direction of solution flow will be opposite to that of the moving car body to be phosphated. In order to ensure consistent good quality of phosphate coating on car body the phosphating bath should be provided with a continuous sludge removal system like filter press to minimize the accumulation of sludge in the phosphating bath. Usually, the sludge containing phosphating bath solution is pumped to Tilted Plate Separator (TPS) where a major portion of the sludge will be separated and collected at the bottom of the separator. The comparatively clear supernatant liquid from the TPS is then pumped through the filter press (containing a series of filters) where the phosphating bath solution will be completely free from sludge and then pumped back to the main phosphating tank. The total sludge separating unit will be under continuous operation as long as the phosphating plant is running and at least 60 to 70 percentages of the filters should always be in working condition for effective sludge removal. Yoshihara et al. [1] recommends a sludge concentration of about $300 \mathrm{ppm}$ maximum at any stage in the phosphating bath.

- The total surface area processed per hour in a given volume of bath solution is very important for maintaining the chemical equilibrium of the phosphating bath. For light and medium coating weight zinc phosphating bath, the optimum recommended rate of processing is about $2 \mathrm{sq}$. $\mathrm{ft} / \mathrm{hour} / 4.5$ liters of bath solution.

- Phosphating solution should be heated indirectly by external plate heat exchanger by using low pressure hot water. The temperature differential between the phosphating solution and hot water should not exceed $10^{\circ} \mathrm{C}$. This will prevent the formation of scale on the heat exchanger plate.

- For high production volume automotive plant, the 
auto dosing system of the chemicals and accelerators (toners) to the phosphating bath is usually recommended to ensure consistently satisfactory phosphate coating on car bodies.

\subsection{Passivating Rinse}

In order to improve the corrosion resistance of phosphate coating on steel surface, it is useful to give a final rinse with chromium containing solutions. This treatment which is known as passivation process provides additional stability to the phosphated surface by partial sealing of the pores in zinc phosphate coating. The trend, so far has been to use passivating solutions containing a mixture of hexavalent and trivalent chromium ions for best results. The latest trend, however, is to use Chrome free formulations for passivation purpose and a number of Zirconia based products are now available for use in automotive finishing industry.

\section{Characterization of Phosphate Coating}

In order to establish the structure-property-performance correlation of the phosphate coating with its protective value and also to carry out failure analysis of the Metal/Phosphate/ED primers interfaces, it is very important to characterize the phosphate coating at microscopic level [6-27]. The key physico-chemical characteristics parameters of phosphate coating on a metal surface are:

- Coatings morphology (crystal size, shape, orientation and coating compactness).

- Crystal phases of the coating.

- Coating weight.

- Coating composition.

- Chemical stability.

Usually coatings morphology and crystal size are determined by Scanning Electron Microscopy (SEM) and the crystal phases are determined by X-ray Diffraction technique (XRD)and elemental analysis on the surface coating may be done by Energy Dispersive X-ray analysis (EDX). Coating weight can be determined by chemical methods by dissolving the coating in dilute chromic acid solution and the phase composition ("P" ratio) may be determined by chemical methods like Atomic Absorption Spectroscopy (AAS) or by XRD techniques [12-21]. The chemical stability of the phosphate coatings may be determined by exposing the coatings in dilute solution of sodium hydroxide and checking the extent of solubility $[19,21]$. The final corrosion performance of phosphated and painted surface maybe evaluated by accelerated tests like salts spray (ASTM-B117) and also by Electrochemical impedance Spectroscopy (EIS] [22-27]. In automotive industry, salts spray tests are widely used to evaluate the performance of phosphated (PT) and Electro-deposited primer (ED) coating. For mild steel surface, the normal specification for anodic electrocoat (AED) process is that the coating system (PT + AED) should pass $600 \mathrm{hrs}$ of salts spray tests (with $20 \mu \mathrm{m}$ primer thickness) while for cathodic electrocoat system (PT + CED) with similar thickness of primer film, the coating system should pass a minimum of $1000 \mathrm{hrs}$ of salts spray test.

The structure, composition and coating weight of phosphate coating deposited on a metal substrate is a function of several factors:

1) Structure and chemical composition of the metal surface.

2) Design of the phosphating chemical.

3) Mode of application i.e. dip or spray.

4) Bath parameters like free acid, total acid, concentration of oxidizing accelerators or toners, temperature, time of coating deposition and loading rate i.e. total surface area processed per hour in a given volume of phosphateing solution.

The effect of some of these parameters on the structure, morphology and performance of phosphate coating on steel surface have been reported in details in some of our earlier works [14,16-19]. In the following section we highlight some key results which are important for the both the development of new phosphating formulations as well as for solving the quality problems encountered during their application to industrial metal finishing process.

\subsection{Morphology and Chemical Composition of Phosphate Coating}

The application of SEM, EDX and XRD techniques provides a comprehensive idea about the physical structure, Chemical composition and nature of the coating deposited on metal surface. The uniformity of crystal size and compactness of the coating is very important for adhesion of the phosphate coating to the metal surface as well as the adhesion of the paint coating or organic coating to the phosphated surface. In Figures 2(a)-(c), the morphology of three different types of phosphate coating deposited on steel surface from three phosphating formulations 1, 2, 3 are shown. The formulations 1 and 2 are conventional high temperature immersion type phosphating formulations $\left(70^{\circ} \mathrm{C}\right)$ with relatively high zinc content 1) and calcium modified zinc phosphating formulations; 2) leading to uniform compact coating with nodular shaped and spherical shaped crystals respectively. In contrast, formulation; 3) which is a tricationic low temperature phosphating formulation $\left(45^{\circ} \mathrm{C}-50^{\circ} \mathrm{C}\right)$ deposits a highly uniform compact coating with cubic shaped crystals. The most important aspect of all these coating is that all three formulations provide highly uniform, thin and compact coating on steel surface but average crystal size varies in the range of from $4-10 \mathrm{mi}-$ 


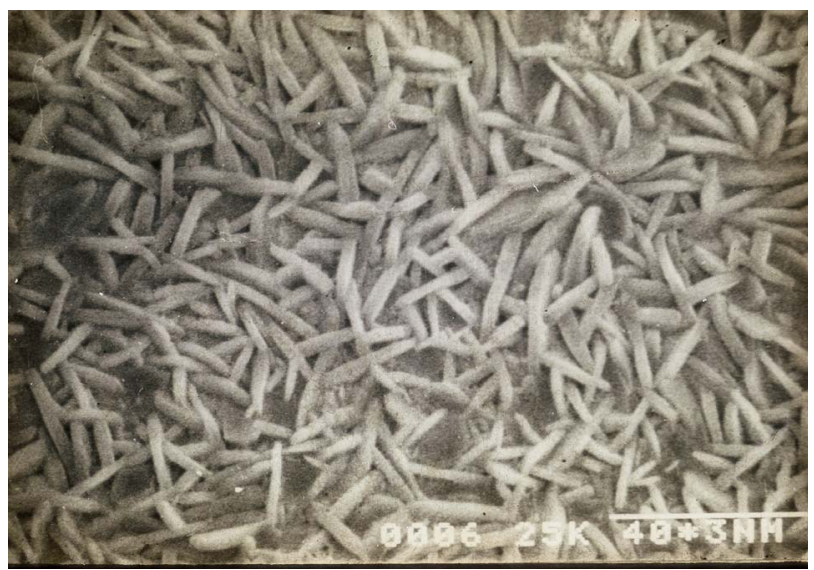

(a)

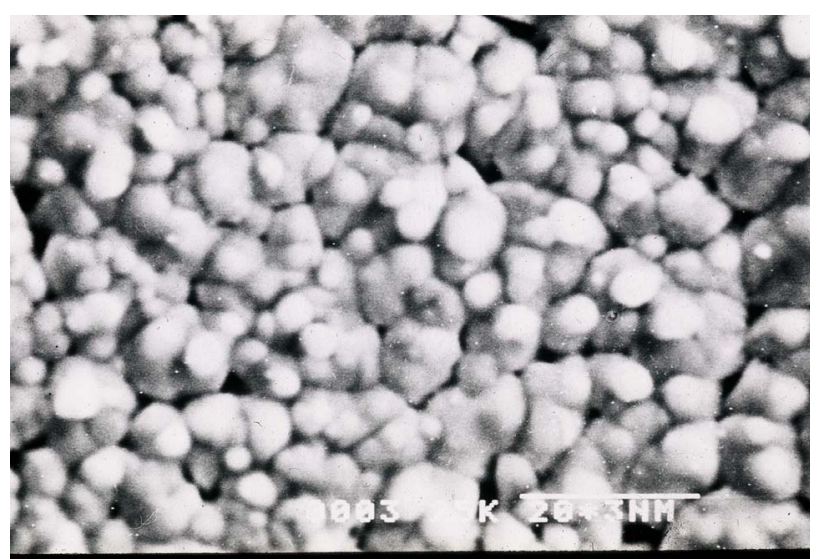

(b)

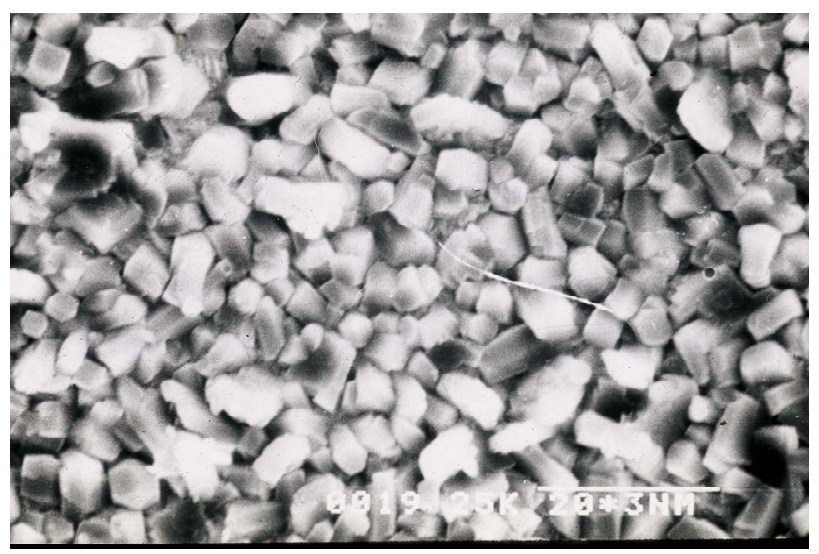

(c)

Figure 2. (a) SEM micrograph of phosphate coating deposited on steel surface (formulation I); (b) SEM micrograph of calcium modified phosphate coating deposited on steel surface (formulation II); (c) SEM micrograph of phosphate coating deposited on steel surface from tricationic phosphating formulation III.

crons for different formulations. Further, the coating composition of all these formulations varies because of difference in the formulations. The corrosion performance of these three phosphating formulations are dis- cussed in the last section. Figure 3 provides an example of poor phosphate coating on steel surface which was not properly cleaned at degreasing stage from formulation III and hence undesirable in production line.

The morphology of phosphate coating on zinc coated steel and aluminium substrates from formulation III are shown in Figures $\mathbf{4}$ and $\mathbf{5}$ respectively. It is quite evident that the coating morphology is very compact on the former but not so satisfactory on aluminium substrate. The XRD diffractograms of phosphate coating on steel and zinc coated steel surface are shown in Figures 6(a) and (b) respectively. It is quite evident that the coating on steel surface consists of both Phosphophyllite and Hopeite phases whereas on zinc coated steel surface the it consists of only Hopeite phase as expected. Similary EDX spectra of phosphate coatings on steel surface from all three formulations are shown in Figure 7. The different elements present in the coating are quite evident from the spectra.

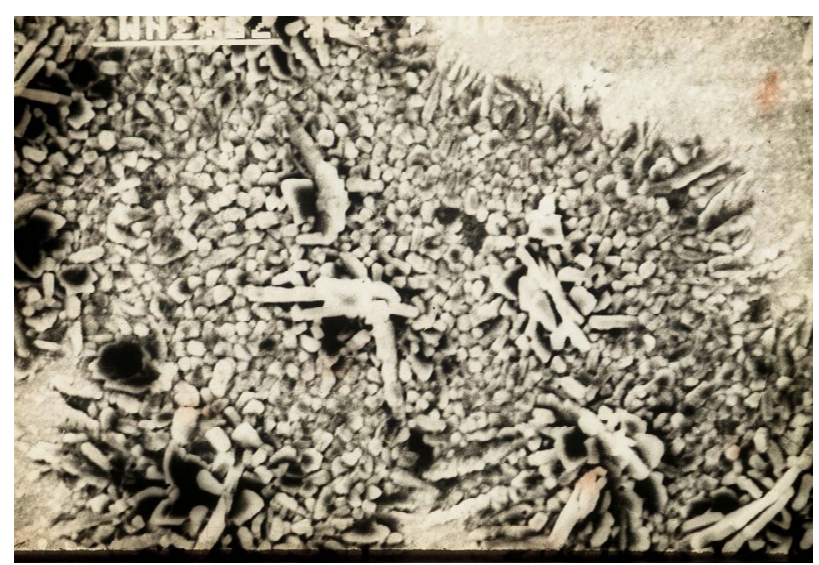

Figure 3. SEM micrograph of poor phosphate coating from formulation III on steel surface which is not properly cleaned.

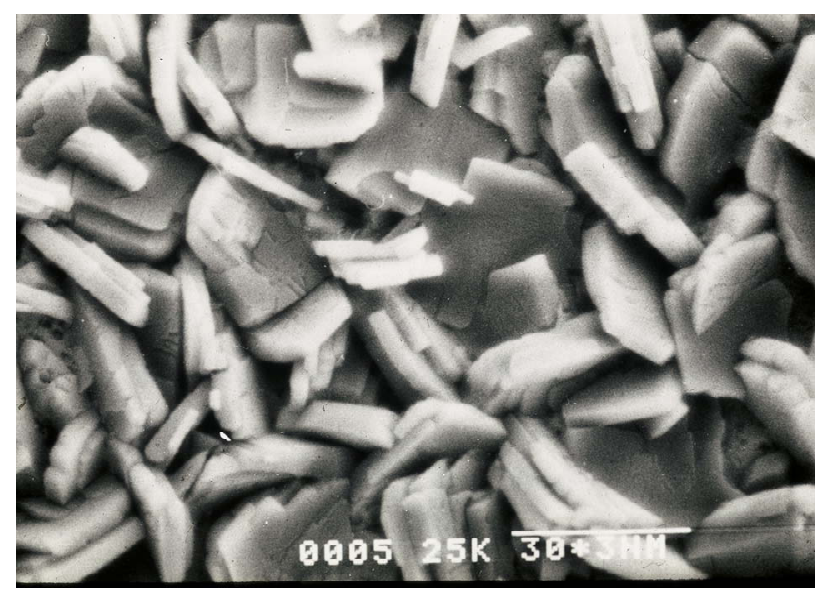

Figure 4. SEM micrograph of zinc phosphate coating deposited on zinc coated steel surface from tricationic phosphating formulation III. 


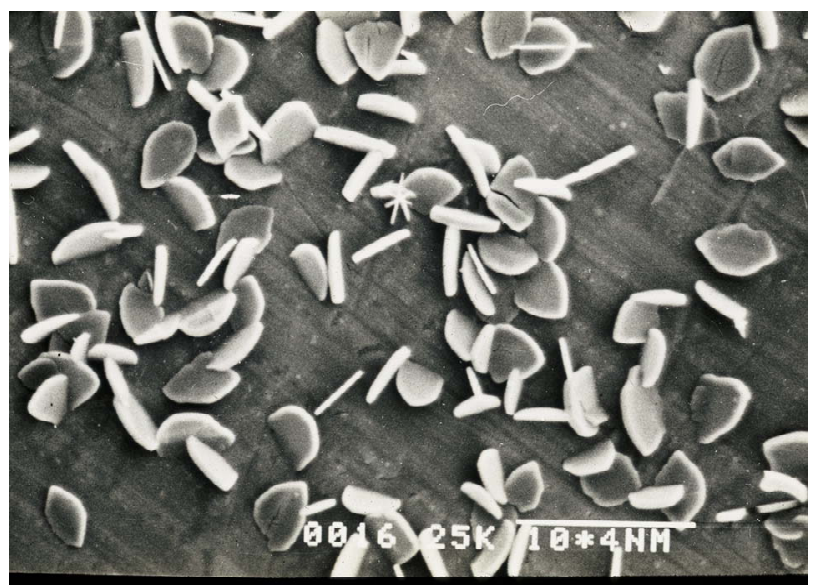

Figure 5. SEM micrograph of phosphate coating on aluminium substrate from tricationic phosphating formulation III.

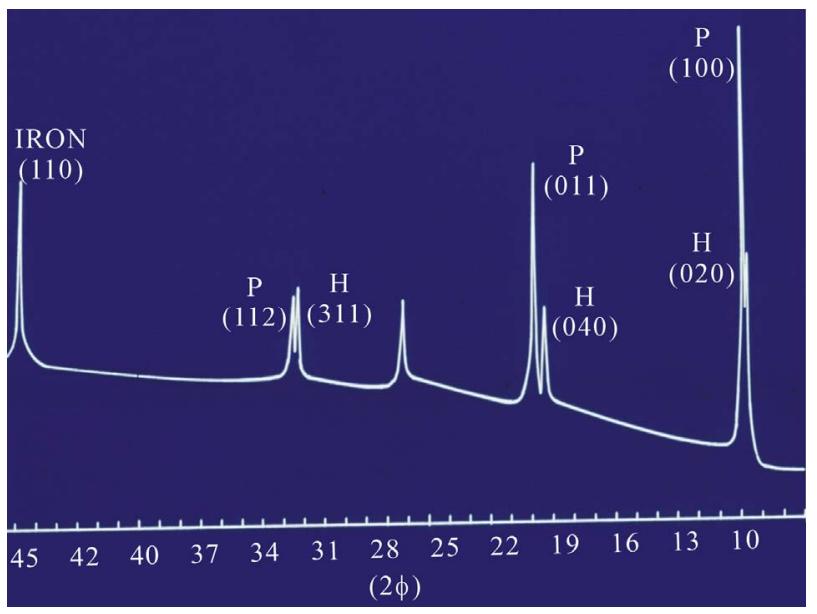

(a)

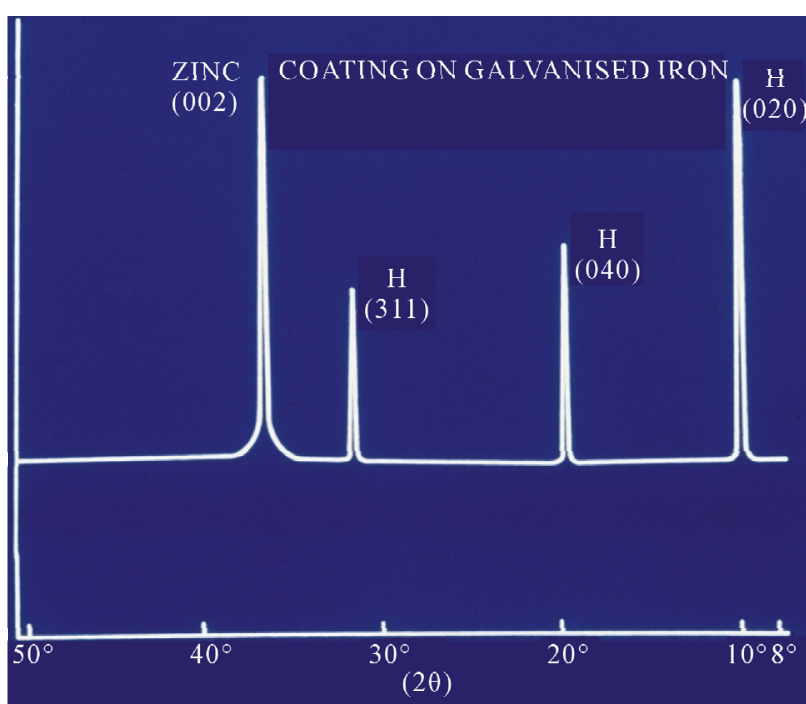

(b)

Figure 6. (a) X-ray diffractogram of phosphate coating on steel surface and (b) X-ray diffractogram of phosphate coating on zinc coated steel surface.

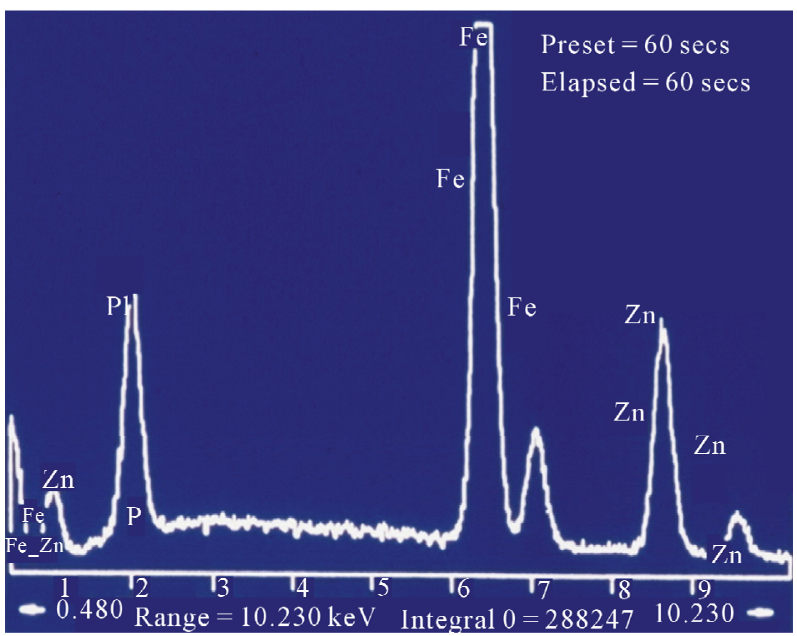

(a)

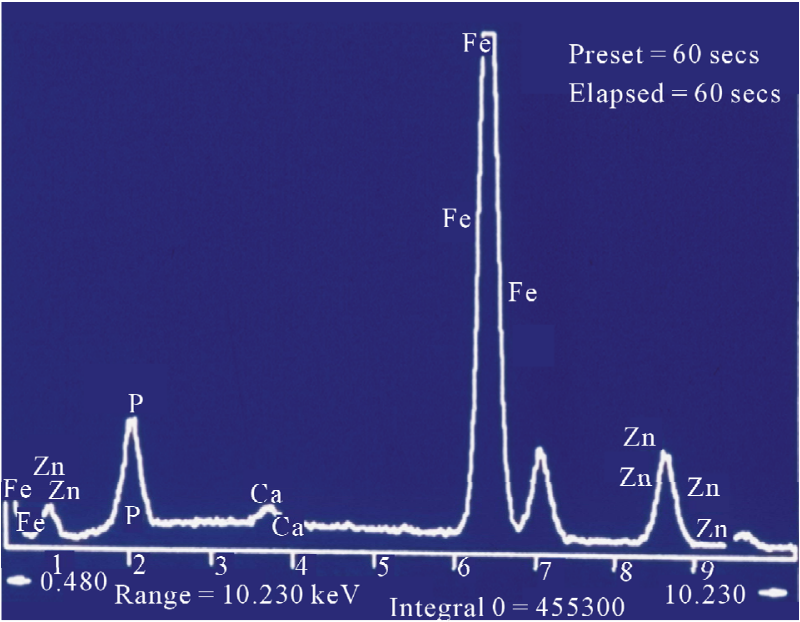

(b)

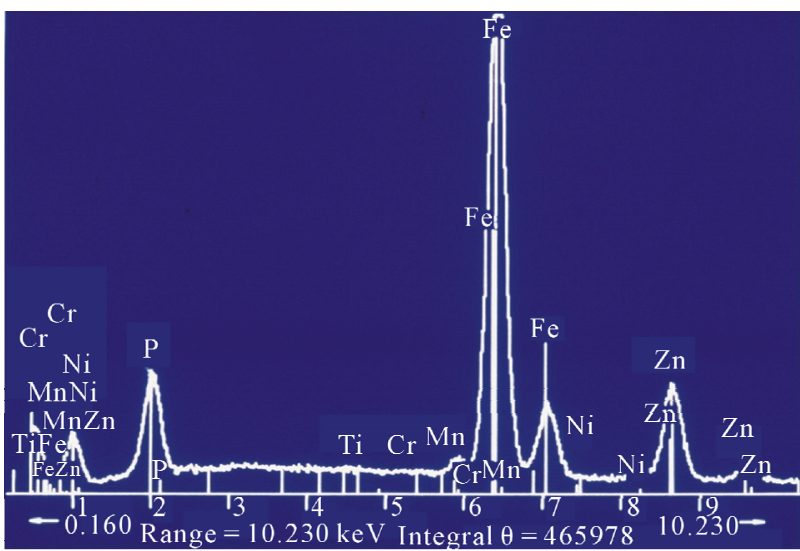

(c)

Figure 7. (a) EDX spectra of phosphate coating from formulation I (b) from formulation II and (c) formulation III.

It may be noted that the phosphate coating layer is the most critical link in the chain of multiple coating layers that are deposited on car body during autobody finishing and thus the integrity of metal/phosphate interface is ex- 
tremely important for better corrosion resistance of car body. An example of excellent steel/phosphate interface is provided by the SEM micrograph shown in Figure 8. The dark part in the micrograph is steel substrate and the bright part is zinc phosphate coating.

\subsection{Effect of Surface Composition on Quality of Phosphate Coating on Steel Surface}

In order to address the problem of variation of coating quality viz. coating morphology and coating weight on steel panels supplied by different steel manufacturers, a systematic work was done on a set of 12 panels procured from different suppliers and phosphated under laboratory conditions and their coating quality was evaluated by SEM technique. The results were classified into four grades A, B, C, D depending on the quality of phosphate coating. Both bulk and surface composition of these samples were determined by Vacuum Emission Spectroscopy and XPS technique respectively and the data revealed that even though the bulk chemical composition of all the panels is essentially quite similar as shown in Table 3, there is substantial difference in the surface composition of the four panels classified under different grades. Figure 9(a) and (b) shows the XPS results (Fe $2 p_{3 / 2}$ and $C 1_{s}$ spectra) of steel surface for four samples $\mathrm{S} 2$, $\mathrm{S} 4, \mathrm{~S} 6$ and $\mathrm{S} 8$. The surface $\mathrm{Fe} / \mathrm{C}$ ratio decreases systematiccally form 0.41 to 0.15 as the coating morphology degrades systematically from the best (A) to the worst (D) as shown in Figures 10(a)-(c) and summarized in Table 4. The SEM picture corresponding to the D-grade steel is not shown here as it is completely amorphous coating without any structure. It was thus established that surface $\mathrm{Fe} / \mathrm{C}$ ratio is a very important index and can be used as a reliable criterion for grading the steel panels and to distinguish the good steel from bad steel as far as the phosphatibility is concerned. More details of this were published in an earlier publication [16].

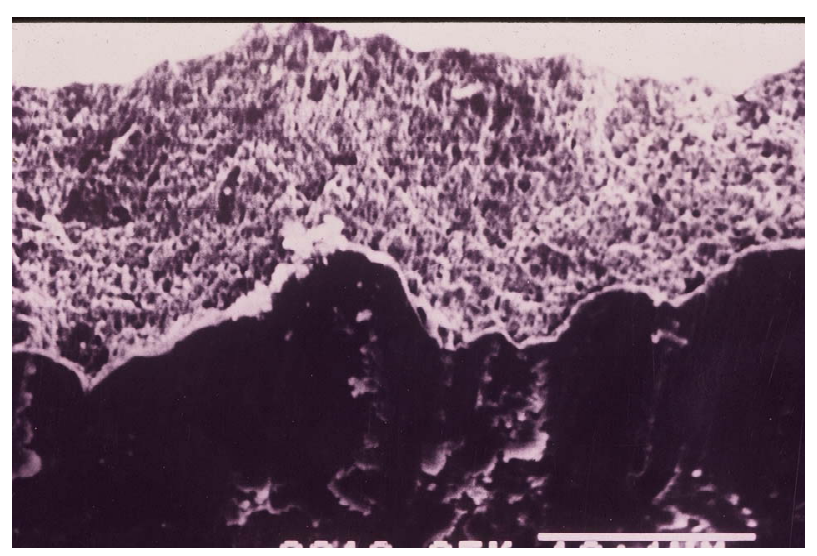

Figure 8. SEM micrograph of steel/phosphate coating interface. Dark part is metal and bright portion s phosphate coating.

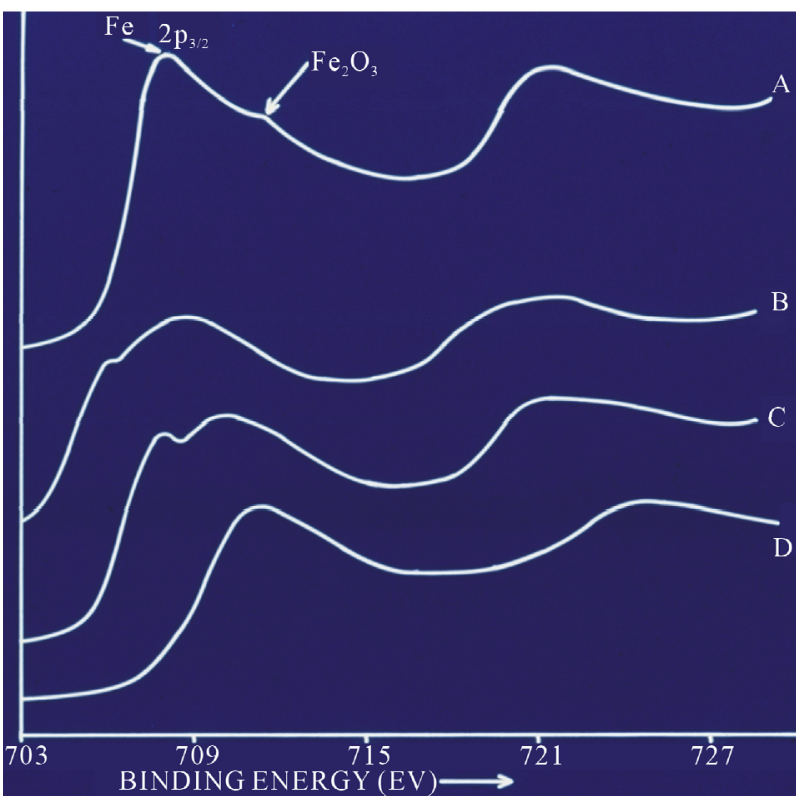

(a)

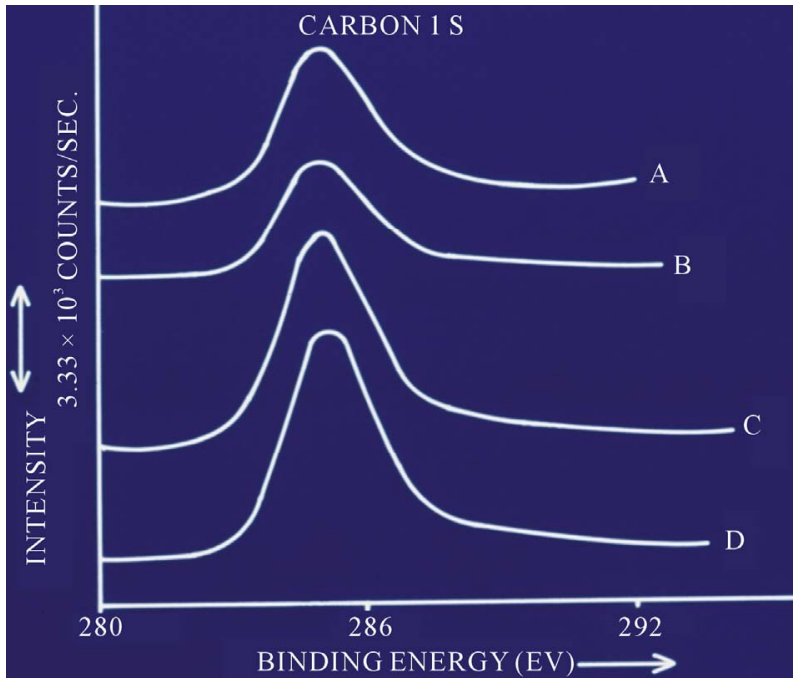

(b)

Figure 9. XPS results of (a) $\mathrm{Fe} 2 \mathrm{p}_{3 / 2}$ (b) $\mathrm{C} 1 \mathrm{~s}$ spectra of steel surface of the four steel samples.

\subsection{Evaluation of Corrosion Performance of Phosphated and Painted Steel Surface}

In order to evaluate the comparative corrosion performance of tricationic phosphating formulation(III) with two conventional immersion type zinc phosphating formulations (I and II), three sets of mild steel panels ( 6 " $\times$ 4 ") were cleaned, phosphated by immersion process at the recommended parameters of the each phosphating formulations in the laboratory, keeping the degreasing, derusting and passivation stages identical. The details of phosphating process parameters for formulations I and II are already reported in earlier work $[17,18]$. The phosphate panels were subsequently coated with an alkyd 
Table 3. Bulk composition of steel samples used in this study.

\begin{tabular}{ccccccccccc}
\hline Sample No. & Elements $\rightarrow \mathrm{Fe}$ & $\mathrm{C}$ & $\mathrm{Mn}$ & $\mathrm{S}$ & $\mathrm{Si}$ & $\mathrm{Ni}$ & $\mathrm{Cr}$ & $\mathrm{Al}$ \\
\hline $\mathrm{S} 2$ & 99.18 & 0.07 & $<0.1$ & 0.024 & 0.01 & 0.04 & 0.11 & $<0.012$ \\
S4 & 98.89 & 0.08 & 0.14 & 0.024 & $<0.01$ & 0.01 & 0.12 & $>0.111$ \\
S6 & 98.77 & 0.059 & $<0.10$ & 0.023 & $<0.01$ & 0.01 & 0.15 & $>0.111$ \\
S8 & 99.37 & 0.153 & 0.26 & 0.022 & 0.05 & 0.01 & 0.16 & $>0.111$ \\
\hline
\end{tabular}

Table 4. Surface analysis of steel samples.

\begin{tabular}{ccc}
\hline Sample No. & Rating of phosphate coating quality & Surface Fe/C ratio \\
\hline S6 & A & 0.41 \\
S4 & B & 0.37 \\
S2 & C & 0.22 \\
S8 & D & 0.15 \\
\hline
\end{tabular}

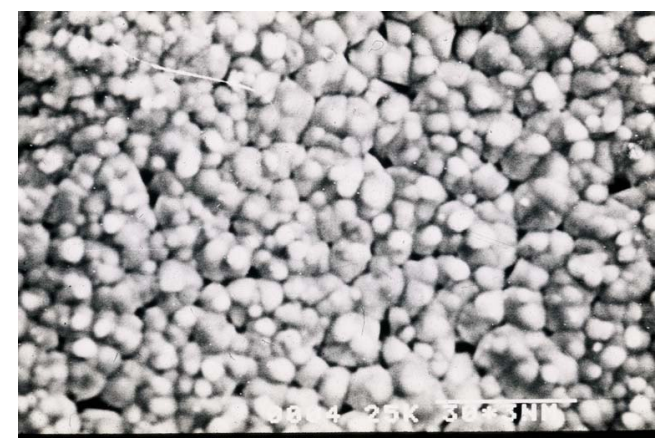

(a)

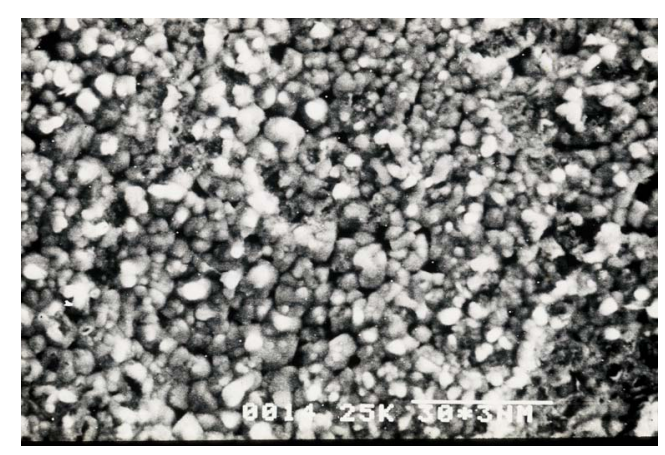

(b)

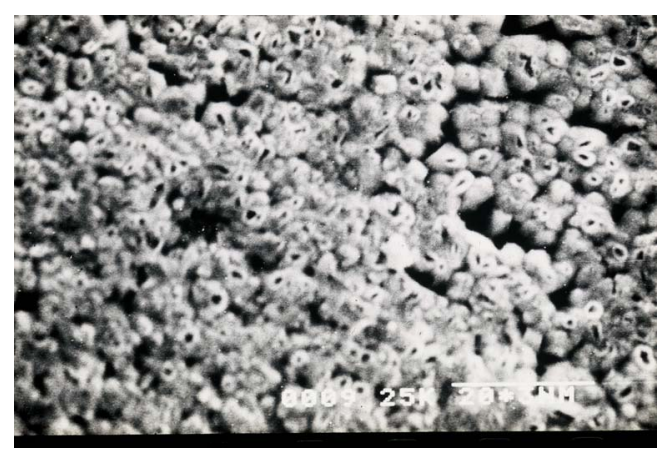

(c)

Figure 10. SEM micrographs of phosphate coating on steel sample (a) S6; (b) S4; (c) S2. based stoving clear to 20 micron thickness by spray process and then baked at $150^{\circ} \mathrm{C}$ for $30 \mathrm{~min}$. The mild steel panels used in this study were procured from an automobile manufacturer and were cut out from a single sheet to minimize the variation on the substrate quality.

$\mathrm{R}_{\Omega}=$ Solution Resistance.

Rpo $=$ Pore Resistance.

Rct $=$ Charge Transfer Resistance.

$\mathrm{Cc}=$ Coating Capacitance.

$\mathrm{Cdl}=$ Double Layer Capacitance.

$\mathrm{Z} \omega=$ Warburg Impedance.

The corrosion performance of the phosphated and alkyd coated panels with20 micron thickness in salts spray test (ASTM-B117) were monitored periodically both visually as well as by Electro-chemical Impedance Spectroscopy (EIS), over a period of $600 \mathrm{hrs}$. The impedance measurements were carried out on these panels at different interval of exposure time over a frequency range of $10^{-2} \mathrm{~Hz}$ to $10^{5} \mathrm{~Hz}$. The amplitude of the signal was $5 \mathrm{mV}$. The impedance measurements were carried out at open circuit potential using a "Schlumberger 1255 Frequency Response Analyzer" (FRA) operated under computer control. The FRA was connected to the electro chemical cell through "EG\&G potentiostat/Galvanostat 273" More details of the experimental set up for impedance measurement have been reported in an earlier publication [26].

In order to interpret the impedance data we have used an equivalent circuit model of painted metal/solution interface as shown in Figure 11. The impedance data were analyzed in terms of three coating parameters viz. Pore resistance (Rpo), coating capacitance $(\mathrm{Cc})$ and breakpoint frequency (fb) [26]. The results of variation of Rpo, $\mathrm{Cc}$ and $\mathrm{f}_{\mathrm{b}}$ as a function of salt spray exposure time are shown in Figures 12-14 respectively. The numerical values of Rpo, Cc and (fb) at $0 \mathrm{hr}, 100 \mathrm{hrs}$ and $300 \mathrm{hrs}$ of salts spray exposure are tabulated in Table 5. A comparison of Rpo values after 300 hrs of salts spray expo- 
Table 5. Variation of Rpo, Cc and $f_{b}$ values of different phosphate coatings on steel surface coated with $20 \mu \mathrm{m}$ thick alkyd coating with salts spray exposure time.

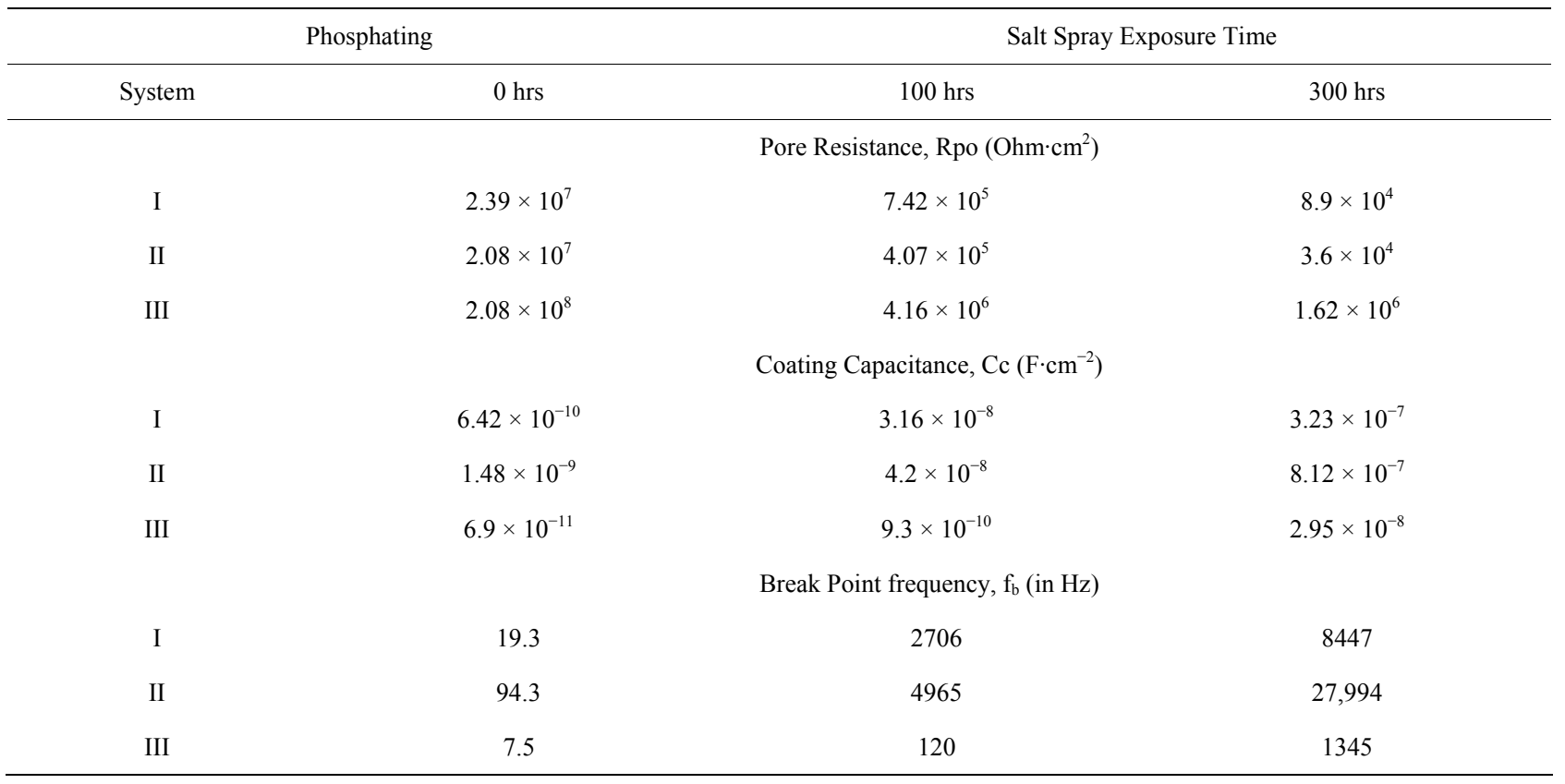

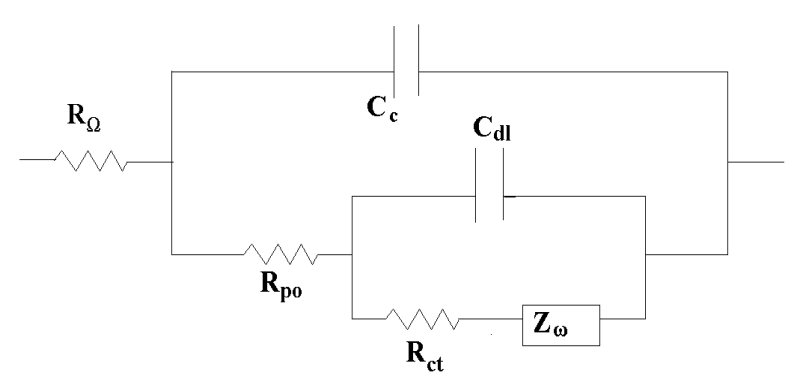

Figure 11. Equivalence circuit model for painted metal/solution interface.

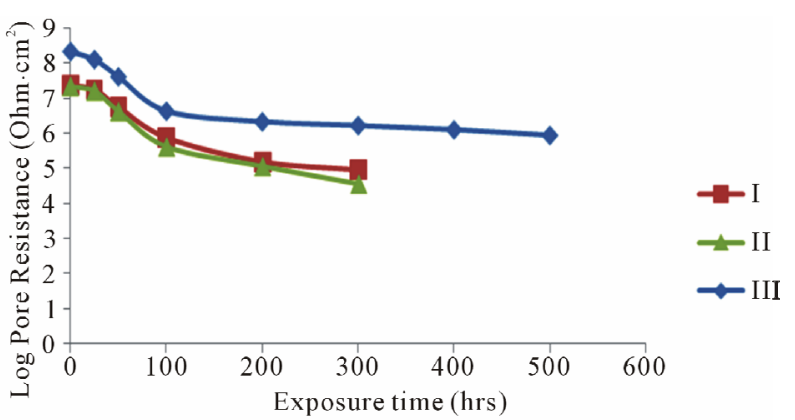

Figure 12. Pore resistance (Rpo) as a function of salt spray exposure time.

sure clearly shows the superior performance of tricationic phosphating formulation III (Rpo-1.62 × $\left.10^{6} \mathrm{Ohm} \cdot \mathrm{cm}^{2}\right)$ compared with formulation I (Rpo- $\left.8.9 \times 10^{4} \mathrm{Ohm} \cdot \mathrm{cm}^{2}\right)$ and formulation II (Rpo-3.6 $\left.\times 10^{4} \mathrm{Ohm} \cdot \mathrm{cm}^{2}\right)$. As shown in Figure 12, the Rpo values for formulation III remain quite steady at this high value even after $500 \mathrm{hrs}$ of salts

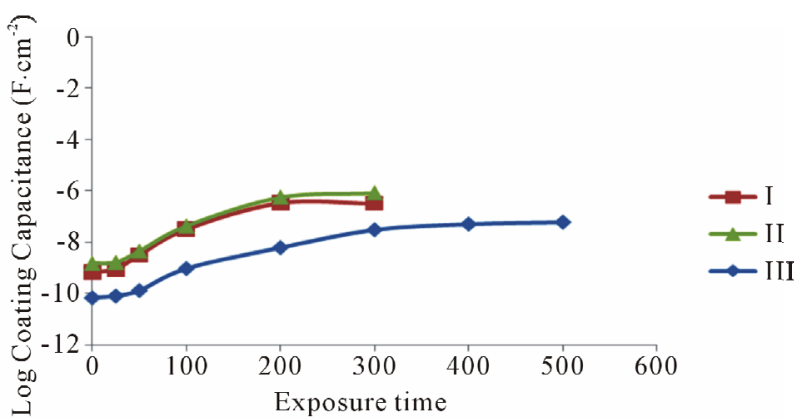

Figure 13. Coating capacitance $(\mathrm{Cc})$ as a function of salt spray exposure time.

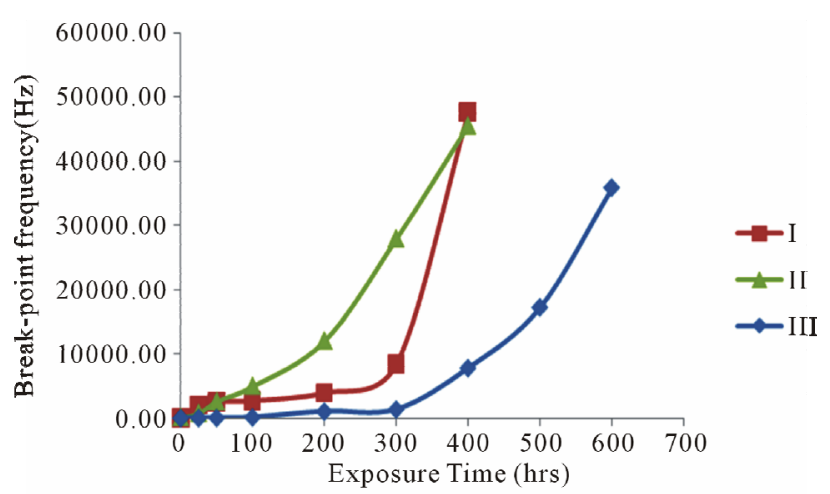

Figure 14. Break point frequency (fb) as a function of salt spray exposure time.

spray exposure, while for other two formulations, Rpo values fall sharply indicating the rapid degradation of the protective value of those phosphate coatings. 
Similarly, Figure 13, where log coating capacitance is plotted against exposure time, indicates that for tricationic formulation (III) the increase in $\mathrm{Cc}$ is relatively much less compared with formulations I and II. The increase in $\mathrm{Cc}$ with exposure time can be attributed to the formation of blisters due to water ingress underneath the film. After a certain exposure time, there was no further increase in the coating capacitance values, either it remained constant or started decreasing. This may be attributed to simultaneous occurrence of two opposing phenomena:

At long exposure times, the ingress of water and accumulation of corrosion products underneath the paint film exert pressure from inside the blister and the blister breaks. This process decreases the capacitance.

2) The nucleation and growth of some blisters continue even at long exposure times. This process increases the capacitance.

The break-point frequency versus exposure time plots (Figure 14) clearly show three distinct stages in coating failure process: water ingress, coating disbonding and blister growth.

Since the break-point frequency is proportional to the area of delamination, the performance of various coatings system could be assessed by comparing the " $\mathrm{f}_{\mathrm{b}}$ " values at a particular exposure time to salts spray environment. For example as shown in Table 5, $\mathrm{f}_{\mathrm{b}}$ value for formulation III after $300 \mathrm{hrs}$. of exposure is $1345 \mathrm{~Hz}$ which is much lower compared with corresponding values $8447 \mathrm{~Hz}$ and $27,994 \mathrm{~Hz}$ for formulation I and II respectively, indicating clearly that formulation III offers much superior corrosion resistance (minimum area of delamination) followed by phosphating formulation I and II, which was also corroborated by visual observation of the panels from salts spray test [26].

The other point to note is the induction times for steep increase in break-point frequency values for this particular coating system (Figure 14) which are approximately 300,150 and 400 hours for phosphating formulations I, II and III respectively which is again a clear indication of the superior adhesion and corrosion performance of phosphating formulation III.

Thus, superior performance of formulation III may be attributed primarily to the difference between chemical composition, compactness and superior alkali resistance of the phosphate coating compared with formulation I and II. The superior alkaline resistance of tricationic phosphating formulation is attributed to the presence of higher level of additional crystal phases like phosphophyllite, phosphomangallite and phosphonicollite besides Hopeite phase in phosphate coating on steel surface.

\section{Acknowledgements}

I would like to thank my collegues Mr. G. N. Bhar and
Mr. P. K. Roy of ICI India, R and D Center for Paints, Kolkata, India for their contribution to this work. I would also like to thank Mr. Nikhilesh Chaudhary of Material Science Department, Indian Association for the Cultivation of Science, Kolkatta, India for all the SEM micrographs and Dr. S. Badrinarayan of National Chemical Laboratory, Pune, India for surface analysis of steel panels and finally to Ms. Gayatri Devi and Prof. V. S. Raja of IIT Mumbai, India for evaluation of phosphate and painted coatings by EIS spectroscopy. Finally, I would also like to thank Miss Vaishali Shinde and my research students, Dr. Shilpa Vaidya, Dr. Priyanka Bhat and Dr. Rohan Jadhav of ICT for putting this paper together in the present form.

\section{REFERENCES}

[1] T. Yoshihara and H. Okita, Transactions of the Iron and Steel Institute of Japan, Vol. 23, 1983, p. 984.

[2] D. B. Freeman, Product Finishing, Vol. 6, 1987.

[3] D. C. Gordan, Corrosion Prevention \& Control, Vol. 7, 1984.

[4] N. Satoh, "Effects of Heavy Metal Additions and Crystal Modification on the Zinc Phosphating of Electrogalvanized Steel Sheet," Surface and Coatings Technology, Vol. 34, No. 2, 1987, pp. 171-181. doi:10.1016/0257-8972(87)90141-1

[5] "Corrosion in the Automotive Industry," Metals HandBook, Vol.13, 9th Edition, p. 1011.

[6] E. L. Ghali and R. J. A. Potvin, "The Mechanism of Phosphating of Steel," Corrosion Science, Vol. 12, No. 7, 1972, pp. 583-594. doi:10.1016/S0010-938X(72)90118-7

[7] Corrosion, Ed. L. L. Shreir, Newnes ButterWorth, London,Vol. 2, 1976, pp. 16:19-16:26

[8] ASM Handbook, "Surface Engineering," ASM International Ohio, USA, Vol. 5, 1996, Phosphate Coatings, pp. 378-404.

[9] ASM Handbook, Corrosion, ASM International, Ohio, USA, Vol. 13 (1996), T. W. Cape, Phosphate Conversion Coating, pp. 383-388.

[10] D. B. Freeman, "Phosphating and Metal Pretreatment," Woodland Faulkner, Cambrige, 1986.

[11] W. Machu, "Handbook of Electropainting Technology," Electrochemical Publications Ltd., UK, 1978.

[12] W. Rausch, "The Phosphating of Metals," AFM International, USA, 1990.

[13] T. S. N. S. Narayanan, "Surface Pretreatment by Phosphate Conversion Coatings-A Review," Reviews on Advanced Materials Science, Vol. 9, No. 2, 2005, pp. 130-177.

[14] N. C. Debnath, Paint India Annual, 1985/86, pp. 19-25.

[15] S. Maeda, Journal of Coatings Technology, Vol. 55, 1983, p. 43.

[16] N. C. Debnath and P. K. Roy, Transactions of the Institute of Metal Finishing, Vol. 74, No. 1, 1996, p. 17. 
[17] N. C. Debnath, G. N. Bhar and S. Roy, Jocca, Vol. 72, 1989, p. 492.

[18] P. K. Roy and N. C. Debnath, Surface Coatings International, Vol. 76, No. 5, 1993, p. 214.

[19] G. N. Bhar, N. C Debnoth and S. Roy, "Effects of Clacium Ions on the Morphology and Corrosion Resistance of Zinc-Phosphated Steel," Surface Coatings International, Vol. 35, No. 1-2, 1988, pp. 171-179. doi:10.1016/0257-8972(88)90066-7

[20] J. P. Servais, B. Schmitz and V. Leroy, Material Performance, Vol. 27, 1988, 56.

[21] W. J. Van Ooij and A. Sabota, Journal of Coatings Technology, Vol. 61, 1989, 778, 51.

[22] B. A. Cooke, "Organic Coatings. Science \& Technology," In: G. D. Parfitt and A. V. Patsis, Eds., Marcel Dekker lnc., New York, Vol. 7, 1984, pp. 197-222.

[23] S. Maeda, T. Asa and M. Yamamoto, Organic Coatings: Science and Technology, Vol. 7, 1984, pp. 223-247.
[24] M. Mansfield, M. W. Kendig and S. Tsai, "Evaluation of Corrosion Behavior of Coated Metals with AC Impedance Measurements," Corrosion, Vol. 38, No. 9, 1982, pp. 478-485. doi: $10.5006 / 1.3577363$

[25] N. Tang, W. J. van Ooij, G. Gorecki, "Comparative EIS Study of Pretreatment Performance in Coated Metals," Progress in Organic Coatings, Vol. 30, No. 4, 1997, pp. 255-263. doi:10.1016/S0300-9440(96)00691-1

[26] V. S. Raja, R. Gayatri Devi, A. Venugopul, N. C. Debnath and J. Giridhar, "Evaluation of Blistering Performance of Pigmented and Unpigmented Alkyd Coatings Using Electrochemical Impedance Spectroscopy," Surface and Coatings Technology, Vol. 107, No. 1, 1998, pp. 1-11. doi:10.1016/S0257-8972(98)00504-0

[27] N. C. Debnath and G. N. Bhar, European Coatings Journal, No. 4, 2002, p. 1. 